\title{
FPGA Dynamic Power Minimization through Placement and Routing Constraints
}

\author{
Li Wang, Matthew French, Azadeh Davoodi, and Deepak Agarwal \\ Information Sciences Institute, University of Southern California, Arlington, VA 22203, USA
}

Received 15 December 2005; Accepted 18 April 2006

\begin{abstract}
Field-programmable gate arrays (FPGAs) are pervasive in embedded systems requiring low-power utilization. A novel power optimization methodology for reducing the dynamic power consumed by the routing of FPGA circuits by modifying the constraints applied to existing commercial tool sets is presented. The power optimization techniques influence commercial FPGA Place and Route (PAR) tools by translating power goals into standard throughput and placement-based constraints. The Low-Power Intelligent Tool Environment (LITE) is presented, which was developed to support the experimentation of power models and power optimization algorithms. The generated constraints seek to implement one of four power optimization approaches: slack minimization, clock tree paring, $N$-terminal net colocation, and area minimization. In an experimental study, we optimize dynamic power of circuits mapped into $0.12 \mu \mathrm{m}$ Xilinx Virtex-II FPGAs. Results show that several optimization algorithms can be combined on a single design, and power is reduced by up to $19.4 \%$, with an average power savings of $10.2 \%$.
\end{abstract}

Copyright (C) $2006 \mathrm{Li}$ Wang et al. This is an open access article distributed under the Creative Commons Attribution License, which permits unrestricted use, distribution, and reproduction in any medium, provided the original work is properly cited.

\section{INTRODUCTION}

Field-programmable gate arrays (FPGAs) now handle most digital signal processing functions in an embedded platform. However, many embedded platforms, such as handheld devices, distributed sensors, and satellites, demand low power in order to increase their functional lifetime. While SRAM-based FPGAs have a short design cycle, steadily decreasing cost, and growing performance, power consumption remains a concern [1]. The trend from one FPGA device family to another is the number of configurable logic blocks (CLBs) and maximum operating frequency scale exponentially, while corresponding decreases in operating voltage have been much slower to arrive, resulting in an exponentially increasing maximum power consumption per device [2]. Therefore, power must be considered at every level, from VLSI issues such as transistor layout and leakage current, to the software that determines how efficiently a user's design is implemented on an FPGA.

There have been many FPGA power reduction approaches addressing different design levels. Several techniques for low power FPGA design have appeared in literature addressing the VLSI design of an FPGA [2-4]. Research has also considered various synthesis-level power optimizations, such as technology mapping to LUT-based FPGAs techniques [5] or reducing glitching power through pipelining [6]. It has also been shown that power can be addressed in the suite of computer-aided design (CAD) algorithms that place and route an end user's circuit onto the FPGA fabric [7].

For our research, we are considering techniques that yield immediate results on today's devices and interoperate with commercial off-the-shelf (COTS) CAD tools. We further restrict our focus to techniques that do not modify the functional behavior of the circuit and guarantee that the user's original timing, or throughput, constraints are met. In this paper, we propose a novel power optimization methodology that converts power optimization goals into constraints compliant with throughput-based COTS PAR tools, minimizing the power consumption of a design's routing interconnect.

In today's FPGAs about $50-70 \%$ of total power is dissipated in the interconnection network [8]. The dynamic power of nets is characterized by

$$
P_{\text {dynamic }}=\sum_{i}\left(C_{i} \times F_{i} \times V^{2}\right),
$$

where $C_{i}$ and $F_{i}$ are the capacitance and average toggle rate of the $i$ th net, and $V$ is the internal voltage. For a given net, the dynamic power can be reduced by diminishing its capacitance, or length. Nets with high toggle rates and/or high capacitance therefore are good potential targets for decreasing the overall power and serve as the motivation of the power optimization schemes presented. 
In this work, we first introduce the Low-Power Intelligent Tool Environment (LITE) created for this research. This environment allows the development and experimentation of power models, tracking dynamic power consumption during simulation, and power estimation at the synthesis level, while providing an infrastructure to rapidly design and execute new power optimization algorithms. Using LITE, four power optimization approaches were created and implemented that generate constraints compliant with the COTS Xilinx PAR tools.

The rest of the paper is organized as follows. In Section 2, we introduce the relevant background on the Xilinx VirtexII FPGA microarchitecture as it pertains to routing interconnects and power consumption. Section 3 addresses the software, first describing the Xilinx CAD tool flow and then the infrastructure of the Low-Power Intelligent Tool Environment (LITE). Section 4 introduces the power optimization algorithms and their experimental results. In Section 5, the results of combining the power optimization methods are presented. In Section 6, we extend our software results to a hardware testbed and validate our approach. Finally, Section 7 concludes the paper.

\section{FPGA DEVICE POWER CHARACTERISTICS}

In order to create efficient power optimization algorithms, the underlying FPGA architecture must be well understood. Though the techniques presented here work for a variety of FPGA microarchitectures, we will limit our focus in this paper to the Xilinx Virtex-II FPGA. The Virtex-II FPGA devices are comprised of input/output blocks (IOBs), located on the edges of FPGA chips, and configurable logic blocks (CLBs) organized as a two-dimensional array inside the ring of IOBs [9]. Each CLB includes four slices and an interconnect block. Slices provide functional elements for combinational and synchronous logic which can be configured as ROMs, LUTs, or SRLs, flip-flops, or other circuitry. The logic of a user's circuit will be considered static after synthesis and capacitance information of each microarchitecture feature can be found in literature [8] or in software by exporting information from Xilinx XPower power analysis tool.

In Virtex-II FPGAs, CLBs connect to the global routing matrix through the interconnect fabric. Global routing resources are comprised of 4 types of lines: long lines, hex lines, double lines, and direct connect lines, in the order of their length. Interconnect capacitance can also be found by exporting results from the Xilinx XPower tool. It is important to note that a net in a user's circuit may have any combination of routing, from carry-chains and internal CLB routing with minimal capacitance, to several vertical and horizontal hops along longer interconnect routes. A quick glance at the interconnect capacitance in Table 1 shows that a reduction by only one interconnect length can yield about a $30 \%$ reduction in capacitance.

The clocking infrastructure is also critical to consider when optimizing power. With 100\% toggle rates and extremely high fanouts, these nets typically consume the most power in a design, even with dedicated clocking lines. The

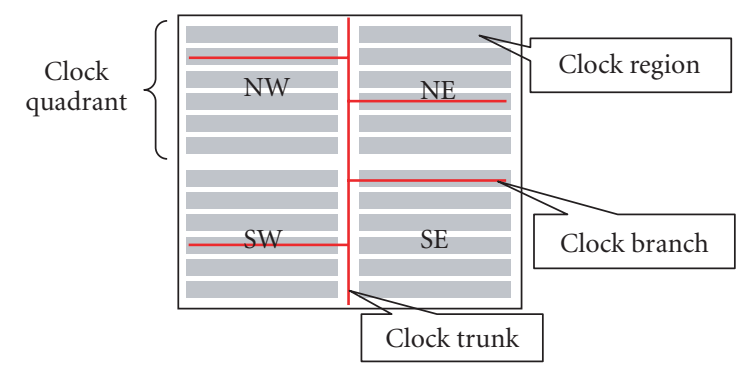

FIGURE 1: Clock tree and clock regions in XC2V6000 FPGA.

TABLE 1: Interconnect capacitance.

\begin{tabular}{lc}
\hline Interconnect line & Capacitance $(\mathrm{pF})$ \\
\hline Direct line & 9.4 \\
Double line & 13.2 \\
Hex line & 18.4 \\
Long line & 26.1 \\
\hline
\end{tabular}

Virtex-II architecture supports 16 clocks, and 8 global clocks can be used in each quadrant of the device. In each quadrant, clocks are organized in clock regions. Figure 1 depicts the clock tree and clock regions in the XC2V6000 FPGA device.

Although we are focusing on the Virtex-II architecture, the algorithms presented here can be adapted to other architectures as well, as long as cost tables such as those in Table 1 are adjusted to account for minor architecture differences.

\section{SOFTWARE INFRASTRUCTURE}

This section discusses the software infrastructure developed to rapidly analyze FPGA power consumption and implement power optimization algorithms. As the developed tools interoperate with the COTS CAD tool flow, the Xilinx PAR tools will be discussed first with respect to power and the LowPower Intelligent Tool Environment (LITE) is described afterwards. Finally, the experiment framework and validation methodology are presented.

\subsection{Xilinx tool flows}

The Xilinx tool flow of design implementation includes the following steps [10].

(i) Translate, which merges the incoming netlists and constraints into a Xilinx design file.

(ii) Map, which fits the design into the available resources on the target device.

(iii) Place and Route, which places and routes the design to the timing constraints.

After Place and Route, the resulting netlist can be input into the Xilinx XPower tool to create a detailed power consumption report. HDL models can be created after PAR for back-annotated simulation to increase the precision of 


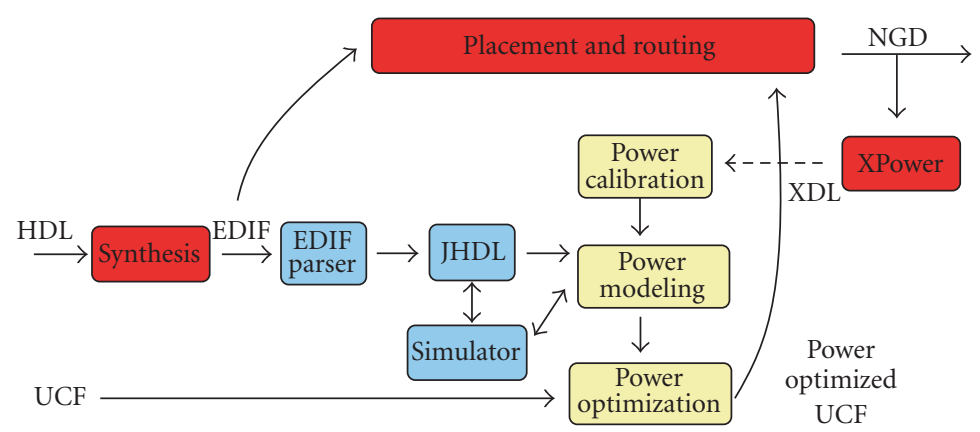

LITE component

JHDL tool

COTS tool

Figure 2: LITE tool flow.

XPower reports. All experiments were run using the Xilinx ISE 6.3 toolset.

\subsection{LITE tool flow}

The Low-Power Intelligent Tool Environment (LITE) was created to facilitate power research by elevating power to a first-order design parameter. It uses calibration, modeling, and estimation techniques to provide automated power estimation at the higher, logic-based EDIF level, where it is easier for a circuit designer to relate the analysis back to their HDL input. In this work, LITE is expanded to incorporate power optimization algorithms that generate UCF file constraints to be passed along to the Xilinx PAR tools as shown in Figure 2.

LITE consists of three components designed to expand the existing COTS power analysis capabilities and experiment with power optimization algorithms: power calibration, power modeling, and power constraint generation. The LITE tool infrastructure is an extension of the JHDL environment. As presented in [11], the JHDL environment provides a high-level tool suite for querying circuit components, running simulations, and tracking signal transitions. LITE builds upon these capabilities to add knowledge about circuit component and interconnect capacitance, monitor a circuit's power consumption during simulation, sort the most power intensive modules within a circuit, and plot various power consumption metrics of the design. A separate EDIF import tool was developed that enables FPGA designs generated by any 3 rd party synthesis tool to be imported into LITE. Simulation results can be obtained by either importing a VCD file or writing a JHDL test bench.

The power calibration component interacts with the Xilinx CAD tools to extract the relevant parameters for power modeling: capacitance, toggle rates, fanout, and power. Xilinx XPower reports contain detailed analysis of placed and routed circuits' power characteristics, and this information can be imported to LITE to obtain the capacitance values of every microarchitectural component, logic element, and interconnect. LITE can then use this information to track and display dynamic power consumption during simulation, or use these values as device power libraries for post-synthesis power modeling and estimation.

The power modeling component allows detailed power analysis of a user's circuit both at the post-synthesis level and the placed and routed level. Post-synthesis power modeling is achieved by combining known logic component capacitance values with routing interconnect length projection techniques developed in [11]. Exact routing capacitances cannot be known until PAR has been completed, however these estimation models are extremely useful in pinpointing power consumption hot spots early on in the design flow and prioritizing nets for power optimization during the PAR process.

By leveraging the JHDL/EDIF infrastructure, this tool suite also enables users to import their designs into the LITE environment, run simulations, track signal transition rates and power consumption over time, as in Figure 3, sort hierarchy modules by power consumption, and cross-probe power overlays with the schematic and waveform viewers inherent to JHDL. Simulations and power analysis can be performed at either the post-synthesis or placed and routed netlist level and allows the direct comparison of the synthesized circuit power against it's placed and routed netlist power.

The power optimization component utilizes the output of the power analysis component to apply the power optimization techniques discussed in Section 4. As mentioned earlier, the power optimization techniques in LITE do not modify design logic, but rather feed additional constraints to the PAR tools such that the existing PAR algorithms can still meet a user's throughput specifications while also reducing power. To support this, the power optimization component is capable of inspecting the area, resources, and size of the targeted FPGA device and the user's circuit, reads in any existing UCF file constraints, and prioritizes the original constraints. 
TABLe 2: Benchmark circuits.

\begin{tabular}{|c|c|c|c|c|c|c|}
\hline Design & Part number & Original timing $(\mathrm{MHz})$ & Signal power (\%) & Logic power (\%) & Clock power (\%) & Baseline power $(\mathrm{mW})$ \\
\hline CRC & $\mathrm{XC} 2 \mathrm{~V} 80$ & 16 & 28 & 42 & 30 & 31 \\
\hline FM & $\mathrm{XC} 2 \mathrm{~V} 250$ & 55 & 43 & 45 & 12 & 102 \\
\hline VGA & $\mathrm{XC} 2 \mathrm{~V} 250$ & $\begin{array}{l}125 \\
133.3\end{array}$ & 18 & 39 & 43 & 138 \\
\hline USBF & XC2V500 & $\begin{array}{l}238 \\
105\end{array}$ & 33 & 30 & 37 & 82 \\
\hline PCI & $\mathrm{XC} 2 \mathrm{~V} 1000$ & 100 & 10 & 33 & 57 & 39 \\
\hline Conv & $\mathrm{XC} 2 \mathrm{~V} 1000$ & 66 & 23 & 55 & 22 & 163 \\
\hline DES3 & XC2V2000 & 100 & 43 & 21 & 36 & 139 \\
\hline Mem & XC2V6000 & $\begin{array}{r}83 \\
160 \\
40\end{array}$ & 8 & 59 & 33 & 643 \\
\hline S1 & XC2V6000 & $\begin{array}{r}180 \\
75 \\
33 \\
33\end{array}$ & 12 & 10 & 78 & 251 \\
\hline S2 & XC2V6000 & $\begin{array}{l}250 \\
100 \\
\end{array}$ & 9 & 12 & 79 & 1020 \\
\hline
\end{tabular}

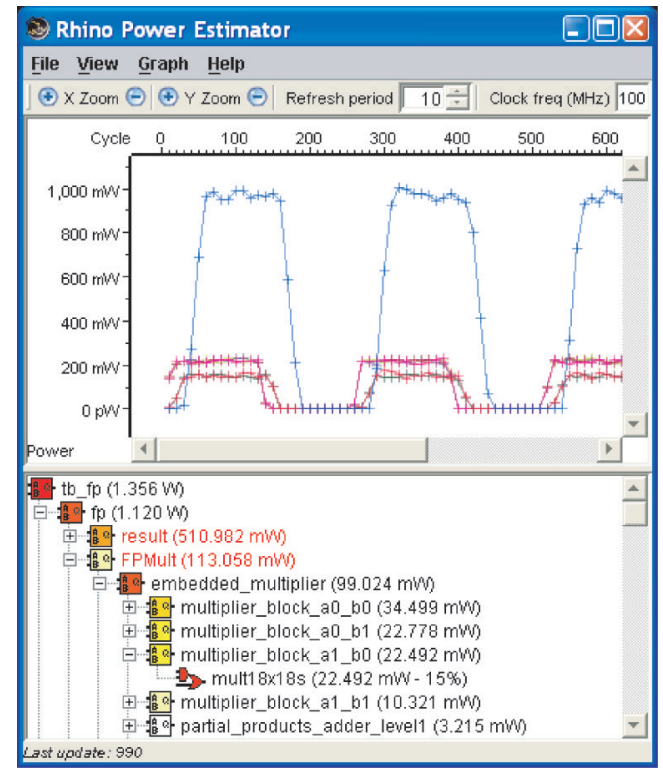

Figure 3: LITE simulation.

\subsection{Experimental framework}

The methodology for power optimization and power verification can also be seen in Figure 2. To perform power optimization, a user imports its design using the EDIF parser, generates a power simulation using the LITE power modeling component, and then generates a new UCF file using the LITE power optimization component. The original, unaltered EDIF file can then be fed through the Xilinx tools using the new constraints file. To measure the results, we use the Xilinx XPower tool with placed and routed netlists and the same value change dump (VCD) simulation data used as inputs in the LITE power simulation stage.

In order to verify the developed power optimization algorithms, a test suite of ten circuit benchmarks was utilized, listed in Table 2. This suite represents a fairly wide taxonomy of applications, from glue logic (Mem) to cores (CRC, FM, VGA, USBF, PCI, and DES3) to end-to-end applications (Conv, S1, and S2), spanning a wide range of device sizes. Each circuit is mapped into the smallest device possible, such that underutilization does not skew results. All designs also had UCF files specifying I/O pin locations and minimum clocking requirements, shown in the 3 rd column. Multiple clocks are represented by multiple entries. Table 2 also shows the breakout of power consumed by signal, logic, and clock elements and reveals that there is a mix of clock dominant, signal dominant, and logic dominant designs. In the final column, the baseline power, the internal dynamic power of each circuit as reported by XPower is shown, that is, the sum of the dynamic power consumed by logic elements, clock nets, and signal nets. Figure 4 shows the slice/IOB utilizations of these designs. Slice occupation ranges from $14 \%$ to $86 \%$, and $\mathrm{IOB}$ occupation from $11 \%$ to $90 \%$, so there is a fair representation of $\mathrm{I} / \mathrm{O}$ bound as well as compute resource bound circuits.

It should be noted that we have spot checked our results on hardware as well. Our power measurement testbed, shown in Figure 5, is comprised of a PCI-DAS1200 ADC which samples the current sensors connected to the isolated internal voltage supply lines on an Osiris board's XC2V6000 device and provides a resolution $2.7 \mathrm{~mA}$. While actual power consumption was difficult to verify due to variables such as room temperature, device fabrication variances, and conservatism inherent in XPower's capacitance reporting, the 


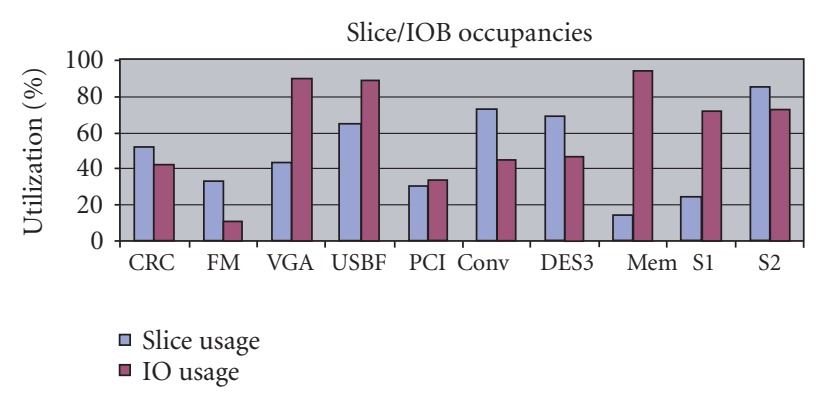

FIgUre 4: Benchmarks slice/IOB utilization.

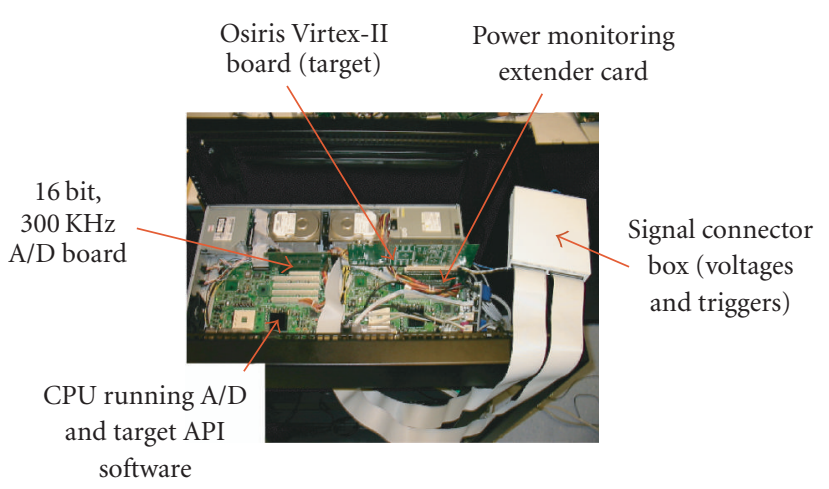

Figure 5: Power measurement testbed.

percentage power reduction between the optimized and baseline versions remained constant between XPower software reports and hardware measurements in experimental testing.

\section{POWER OPTIMIZATION TECHNIQUES}

The power optimization techniques developed center around the theme of creating timing and placement constraints that interoperate with existing COTS PAR tools in order to preserve a user's throughput specifications while also reducing power consumption. The timing and placement constraints influence the COTS tools to use shorter, lower capacitance interconnects. In this paper we provide an overview of four power optimization techniques that each utilizes a different constraint type to enact power optimization. The following subsections explain each technique and present the experimental results achieved.

\subsection{Clock tree paring}

For our first technique, we will focus on trying to reduce the amount of power utilized by the clock nets. As Table 2 shows, even though these nets utilize dedicated, specialized circuitry within the FPGA, these few nets can contribute with $12 \%$ to $79 \%$ of the overall power consumption of a design. This is due to the inherent high toggle rate, high fanout to hundreds or thousands of synchronous logic elements, and long interconnects that span a data path from input to output often across the entire device.

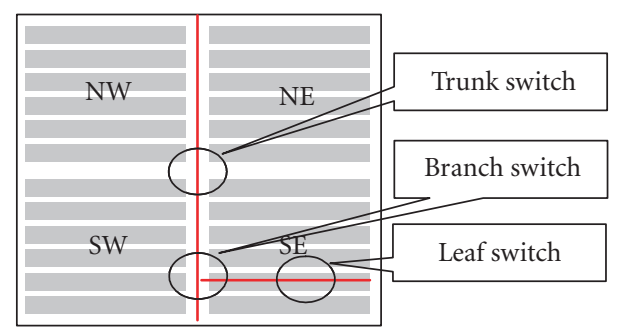

FIGURE 6: Clock net switch types.

The clock tree paring algorithm targets the clock power by utilizing placement constraints to minimize the size of the clock net tree utilized. As introduced in Section 2, in the Xilinx Virtex-II FPGAs, clock nets are distributed on dedicated routing resources. Through FPGA editor and experimentation, we observe that clock network is like a tree, with the main trunk traveling north to south in the middle of the chip, and branches extending west and east into clock regions. The number of clock regions varies depending on the size of the device. The clock tree is gated such that completely unused branches of the tree are effectively turned off. Therefore by placing logic closer together, clocking power can be reduced by gating more of the branches of the clock tree.

From our analysis, we found that there were three types of gating switches, shown in Figure 6, which we will call the trunk switch, branch switch, and leaf switch. The trunk switch is located at the center of the chip. This type of switch is used for turning on or off the upper- or lower-half of the main clock trunks. When a clock net comes into the chip from an input port or digital clock manager (DCM), it goes to the center of the switch-fabric to be routed to the north, or south, or both. Figure 7(a) shows two clock nets as the examples: the clock net on the left is switched to both the upper- and lower-half of the chip. The clock net on the right is switched to the upper-part of the chip only. Figure 7(b) depicts a branch switch. Each Virtex-II has multiple branch switches, and the number varies depending on the size of the device. The switches are located on the path of the main clock trunks. They are responsible for transmitting the clock signals to the clock regions. The clock wire shown in Figure 7(b) travels to both the left and right. The leaf switch is depicted in Figure 7(c). As shown in Figure 7(d), a clock net in the clock region includes a major branch and many subbranches that connect to slices. The leaf switch turns on/off these subbranches. By placing the flip-flops closer to each other, clocking power can be reduced by leaving more branch/subbranch turned off.

The clock tree paring algorithm analyzes a user's circuit, computes a minimum bound to contain all the logic associated with a clock net, and generates area constraints to specify where the associated clock logic may be placed. The area constraint is rectangular, stretching north to south around the clock main trunk. The size of the area is proportional to a clock's fanout. For multiple clock cases, the LITE power analysis component is used to prioritize clocks with higher-power consumption and place them closer to 


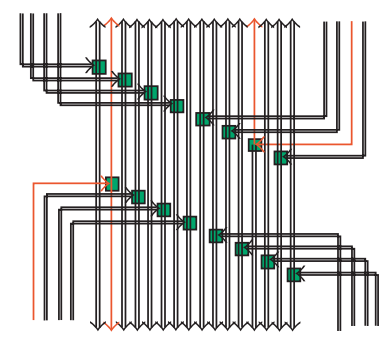

(a)

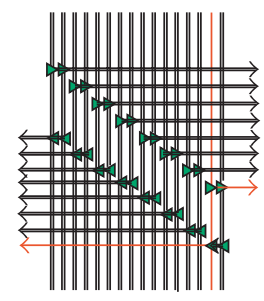

(b)

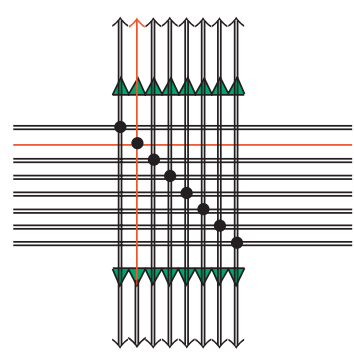

(c)

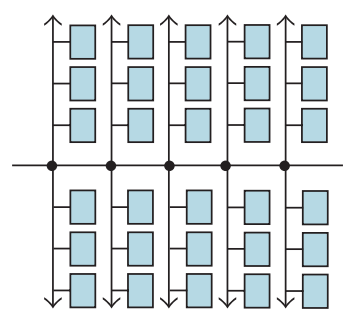

(d)

Figure 7: (a) Trunk switch; (b) branch switch; (c) leaf switch; (d) clock net connected with FFs within a clock region.

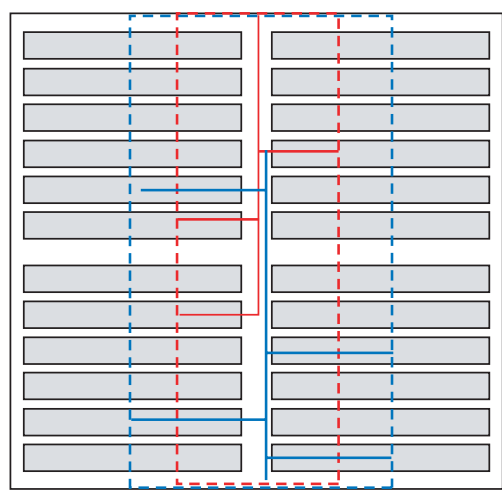

FIGURE 8: Clock area constraints.
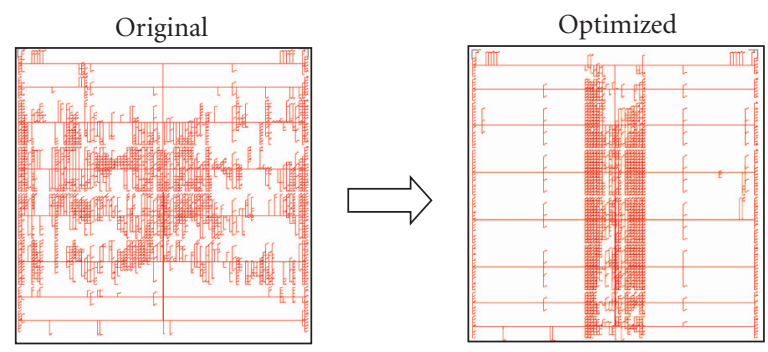

FIgURE 9: Clock area optimization in S1.

the clock trunk, as depicted in Figure 8. It should be noted that the clock groups do not have to be placed radially to the main trunk to save power. Clock power savings, especially in larger designs, come from clustering groups of flip-flops to minimize the number of leaf switches that are activated. In the cases that $\mathrm{I} / \mathrm{O}$ timing is critical, flip-flop clusters can be placed between the I/O pins and a central flip-flop mass about the clock trunk, to pipeline and better preserve timing constraints while also minimizing power. Figure 9 shows an illustrative example of the distributions of one of the clock trees in S1 before and after the clock optimization.

Table 3 shows the results for clock tree paring power optimization. It is interesting to note that even though the signal power increases in several cases, the clock power savings
TABLE 3: Clock tree paring results.

\begin{tabular}{l|rccc}
\hline Design & $\begin{array}{l}\text { Signal } \\
\text { power } \\
\text { reduction }\end{array}$ & $\begin{array}{l}\text { Logic } \\
\text { power } \\
\text { reduction }\end{array}$ & $\begin{array}{l}\text { Clock } \\
\text { power } \\
\text { reduction }\end{array}$ & $\begin{array}{l}\text { Total } \\
\text { power } \\
\text { reduction }\end{array}$ \\
\hline CRC & $3.6 \%$ & $0.0 \%$ & $16.4 \%$ & $5.9 \%$ \\
FM & $-3.0 \%$ & $0.0 \%$ & $36.0 \%$ & $2.9 \%$ \\
VGA & $6.4 \%$ & $0.0 \%$ & $26.5 \%$ & $12.5 \%$ \\
USBF & $2.1 \%$ & $0.0 \%$ & $26.8 \%$ & $10.7 \%$ \\
PCI & $-5.1 \%$ & $0.0 \%$ & $34.2 \%$ & $18.7 \%$ \\
Conv & $4.0 \%$ & $0.0 \%$ & $18.2 \%$ & $4.9 \%$ \\
DES3 & $-4.0 \%$ & $0.0 \%$ & $29.2 \%$ & $8.6 \%$ \\
Mem & $-11.2 \%$ & $0.0 \%$ & $5.1 \%$ & $0.7 \%$ \\
S1 & $-59.9 \%$ & $0.0 \%$ & $11.1 \%$ & $10.7 \%$ \\
S2 & $-13.8 \%$ & $-0.1 \%$ & $28.7 \%$ & $19.4 \%$ \\
\hline
\end{tabular}

are dominate and almost all benchmarks show significant overall power improvement by using this approach. As can be expected, the test circuits not responding as well to this approach (Mem, FM, Conv, and CRC) are considered logic power dominant designs according to Table 2. The clock power dominant designs (S2, PCI, VGA, S1, and USBF) are much more responsive. It should also be noted that though Figure 9 depicts a circuit with low device utilization for illustrative purposes, the efficacy of this technique is more a function of a circuit being clock power dominant than highor low-logic utilization. For example, S2, a clock power dominant circuit, achieves the most significant power reduction with a more than $80 \%$ device utilization, while Mem, the lowest device utilization circuit in our test suite, yields the least significant results.

\subsection{N-terminal net colocation}

$N$-terminal net colocation power optimization is targeted to reduce the power consumed by signal nets. "Terminal" is defined as the sum of the fanin and fanout of a net. For a simplified case, a 2-terminal net is a net with a single fanout. $N$-terminal net colocation restricts net terminals to be placed in adjacent slices. As depicted in Figure 10, net terminals are grouped in pairs, and for each pair, a constraint is used to restrict the two terminals to be located close to each other, and thus reducing the signal net length and power. From our 


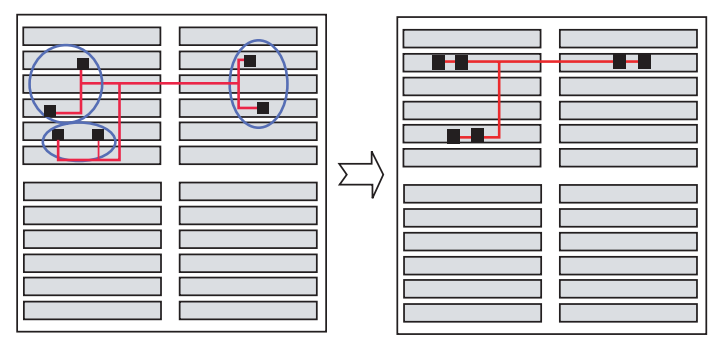

Figure 10: $N$-terminal placement.

LITE calibration and analysis studies, we found that the Xilinx Virtex-II architecture has an east-west bias, meaning that direct connection interconnected in the east-west direction has less capacitance than direct connections in the northsouth direction, sometimes by a factor of up to $50 \%$. So, this algorithm is further enhanced to take advantage of this particular microarchitecture design by prioritizing east-towest relative placement constraints. This algorithm can be updated to reflect other FPGA architecture features as well. The nets are sorted and prioritized by power consumption based on simulations using the LITE power analysis environment to target high-capacitance and high toggle rate nets. In high fanout cases where nets may belong to multiple terminal groups, only the highest priority constraint is created.

Initial experimentation showed that this technique worked well on some nets, however some nets that would naturally be mapped by the COTS PAR tools to low capacitance lines such as carry chains and internal slice nets were now being routed on higher capacitance routing interconnect lines due to the constraints. To avoid this, the algorithm was enhanced to analyze the circuits and selectively avoid putting constraints on certain nets. Several rules were developed to avoid overconstraining the designs as follows.

(i) Avoid nets that are a part of shift registers as the Xilinx slice contains low capacitance, dedicated connection between shift registers that are naturally used by the PAR tools.

(ii) Avoid nets that are a part of carry-chains. The Virtex-II architecture uses dedicated low capacitance carry logic to cascade function generators and provide fast arithmetic addition and subtraction.

(iii) Avoid nets that are mapped internally to slices as these are also low capacitance routes. These nets can be identified as those between look-up tables (LUTs) and multiplexers, and between LUTs and inverters.

The results for the $N$-terminal net colocation algorithm are depicted in Table 4 . Here, we see that the overall power savings is negligible and in a few cases actually becomes worse. The nonzero values in the logic power reduction column show that in some cases slices are being packed more efficiently as desired, however in some designs the $N$-terminal approach causes ripple effects in unconstrained nets, causing more slices to be utilized. While the constrained nets are reduced, other nets belonging to multiple terminal groups may be bumped out of internal slice mappings. Comparing
TABLE 4: $N$-terminal placement results.

\begin{tabular}{l|cccc}
\hline Design & $\begin{array}{l}\text { Signal } \\
\text { power } \\
\text { reduction }\end{array}$ & $\begin{array}{l}\text { Logic } \\
\text { power } \\
\text { reduction }\end{array}$ & $\begin{array}{l}\text { Clock } \\
\text { power } \\
\text { reduction }\end{array}$ & $\begin{array}{l}\text { Total } \\
\text { power } \\
\text { reduction }\end{array}$ \\
\hline CRC & $-9.8 \%$ & $0.0 \%$ & $0.0 \%$ & $-2.9 \%$ \\
FM & $-0.9 \%$ & $-0.5 \%$ & $1.6 \%$ & $-0.4 \%$ \\
VGA & $1.8 \%$ & $0.2 \%$ & $0.6 \%$ & $0.7 \%$ \\
USBF & $-9.2 \%$ & $0.4 \%$ & $-4.2 \%$ & $-4.5 \%$ \\
PCI & $2.6 \%$ & $0.1 \%$ & $-6.8 \%$ & $-3.8 \%$ \\
Conv & $1.6 \%$ & $0.0 \%$ & $-3.4 \%$ & $-0.4 \%$ \\
DES3 & $1.2 \%$ & $0.0 \%$ & $-3.3 \%$ & $-0.7 \%$ \\
Mem & $9.1 \%$ & $0.0 \%$ & $-1.8 \%$ & $0.4 \%$ \\
S1 & $-10.1 \%$ & $0.3 \%$ & $-0.5 \%$ & $-0.6 \%$ \\
S2 & $-1.6 \%$ & $-1.4 \%$ & $1.6 \%$ & $1.0 \%$ \\
\hline
\end{tabular}

the signal power, clock power, and total power columns is insightful as well. For a few circuits, CRC, USBF, and S1, there is a significant reduction in signal power. Closer inspection revealed that these circuits had relatively few high fanout nets. In all cases however, clock power is still dominating and is the main influence on total power.

\subsection{Area minimization}

Another approach to reducing signal power was area minimization. The area minimization power optimization technique is based on the observation that routing interconnect lengths highly depend on the placement of components. By prioritizing the location in favor of power, high capacitance signal lines with high fanout or high transition rates can be grouped together to minimize the power consumed on long interconnects. Constraining the area also has the added affect of trimming the clock tree; however in this case the total area is constrained and clock tree pruning is a residual affect.

This technique is expected to work well on circuits that underutilize the logic available on the chip due to I/O bound designs or poor device size selection. In these designs, the COTS PAR tools place the circuits loosely over the whole chip, doing the minimum to meet the user's timing requirements, as it was designed to do. This behavior however causes longer connection wires and hence increases the total net power. By using area minimization constraints, a design is compacted more tightly in a given area of a chip. Net lengths are shortened and thus power is saved. In an effort to balance the north-south bias of the clock trunk with the eastwest bias of the direct connect signal wires, a rectangular area placed at the center of a chip, with sides proportional to the chip dimensions, is utilized. The size of the area is estimated by analyzing and computing the slice count that each design element needs.

Figure 11 shows an example of the results. On the lefthand side, the circuit is placed loosely over the chip. After using the area minimization power optimization, the circuit is tightly located in an area at the center. It is worth mentioning that eventhough area minimization may have the same effect on the placement of logic components as clock power 


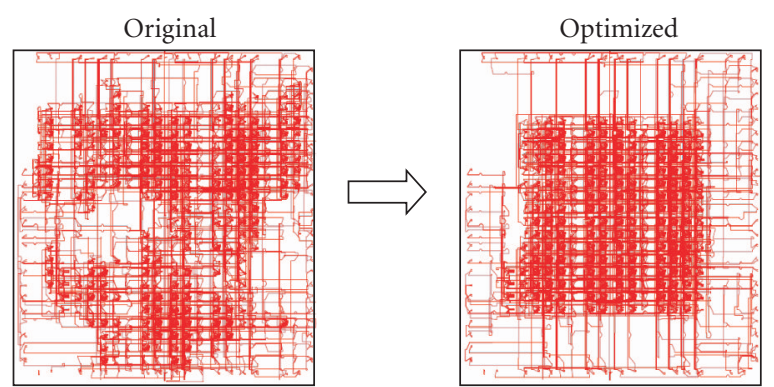

FIgUre 11: Area minimization in VGA.

optimization does, it utilizes different constraints. The clock tree paring technique constrains the clock routing area, influencing the placement of all the logic elements driven by the clock. The area minimization technique explicitly restricts the placement of all components, clocked or nonclocked.

The results of area minimization approach are shown in Table 5, with all circuits showing a positive power reduction. On closer examination the power savings mostly come due to clock power reductions, due to residual clock tree minimization effects similar to those developed in the clock tree paring technique. This technique was unable to be used on the S2 circuit, as this design occupies $87 \%$ of an XC2V6000 device and the area cannot be further minimized.

\subsection{Slack minimization}

Finally, the slack minimization technique seeks to optimize the power on signal nets by tightening timing constraints on power critical nets. The slack minimization algorithm assumes that the PAR tools will leave each net at or just under the user's specified timing requirements, in many cases leaving slack, or extra net length that could be further tightened to reduce capacitance. For this algorithm slack is defined as

$$
\text { Slack }=T_{\text {Spec }}-T_{\text {Logic }}-T_{\min w r},
$$

where $T_{\text {Spec }}$ is the user's timing specification, $T_{\text {Logic }}$ is the timing delay of any combinatorial logic in between flip-flops on the net, and $T_{\min w r}$ is the minimal wire timing delay. For example, in the left-hand side of Figure 12, a flip-flop to flipflop path has two intermediate components, with $1 \mathrm{~ns}$ and 2 ns individual delay. The user's specified clock is running at $100 \mathrm{MHz}$, that is, $10 \mathrm{~ns}$ in period. Therefore, the slack of the path is $7 \mathrm{~ns}$. Without additional constraints, the PAR tools will typically meet the maximum delay necessary to still meet the constraints as it should, creating a wire delay of up to $7 \mathrm{~ns}$. If we allow 1 ns delay between each logic element, we can reduce the interconnect length to $3 \mathrm{~ns}$ and reduce the interconnect capacitance.

The slack minimization technique uses the LITE analysis component to prioritize high capacitance, high toggle rate nets, calculate the slack, and tighten the timing constraints on these nets allowing for only minimal wire length. In practice, nets with ample slack are typically those with two or less levels of combinational logic between flip-flops.

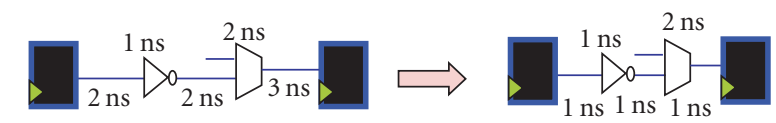

FIGURE 12: Slack minimization.

TABle 5: Area minimization results.

\begin{tabular}{l|cccc}
\hline Design & $\begin{array}{l}\text { Signal } \\
\text { power } \\
\text { reduction }\end{array}$ & $\begin{array}{l}\text { Logic } \\
\text { power } \\
\text { reduction }\end{array}$ & $\begin{array}{l}\text { Clock } \\
\text { power } \\
\text { reduction }\end{array}$ & $\begin{array}{l}\text { Total } \\
\text { power } \\
\text { reduction }\end{array}$ \\
\hline CRC & $-3.1 \%$ & $0.0 \%$ & $6.9 \%$ & $1.2 \%$ \\
FM & $-7.7 \%$ & $0.0 \%$ & $31.6 \%$ & $0.4 \%$ \\
VGA & $-0.7 \%$ & $0.0 \%$ & $1.3 \%$ & $0.4 \%$ \\
USBF & $-1.6 \%$ & $0.0 \%$ & $2.0 \%$ & $0.2 \%$ \\
PCI & $2.3 \%$ & $0.0 \%$ & $1.1 \%$ & $0.6 \%$ \\
Conv & $3.2 \%$ & $0.0 \%$ & $1.9 \%$ & $1.2 \%$ \\
DES3 & $-7.7 \%$ & $0.0 \%$ & $28.8 \%$ & $6.9 \%$ \\
Mem & $13.3 \%$ & $0.0 \%$ & $1.4 \%$ & $1.6 \%$ \\
S1 & $-38.0 \%$ & $0.0 \%$ & $3.0 \%$ & $2.8 \%$ \\
S2 & $\mathrm{NA}$ & $\mathrm{NA}$ & $\mathrm{NA}$ & $\mathrm{NA}$ \\
\hline
\end{tabular}

TABLE 6: Slack minimization results.

\begin{tabular}{l|cccc}
\hline Design & $\begin{array}{l}\text { Signal } \\
\text { power } \\
\text { reduction }\end{array}$ & $\begin{array}{l}\text { Logic } \\
\text { power } \\
\text { reduction }\end{array}$ & $\begin{array}{l}\text { Clock } \\
\text { power } \\
\text { reduction }\end{array}$ & $\begin{array}{l}\text { Total } \\
\text { power } \\
\text { reduction }\end{array}$ \\
\hline CRC & $0.9 \%$ & $0.0 \%$ & $0.0 \%$ & $0.3 \%$ \\
FM & NA & NA & NA & NA \\
VGA & $-1.7 \%$ & $0.0 \%$ & $-1.0 \%$ & $-0.7 \%$ \\
USBF & $0.0 \%$ & $0.0 \%$ & $-2.0 \%$ & $-0.7 \%$ \\
PCI & $2.4 \%$ & $0.0 \%$ & $-0.4 \%$ & $0.0 \%$ \\
Conv & $-0.6 \%$ & $0.0 \%$ & $1.2 \%$ & $0.1 \%$ \\
DES3 & $-0.7 \%$ & $0.0 \%$ & $-3.8 \%$ & $-1.7 \%$ \\
Mem & $14.4 \%$ & $0.0 \%$ & $2.8 \%$ & $2.1 \%$ \\
S1 & $-15.3 \%$ & $0.0 \%$ & $-0.8 \%$ & $-0.9 \%$ \\
S2 & $-1.6 \%$ & $0.1 \%$ & $5.4 \%$ & $4.1 \%$ \\
\hline
\end{tabular}

The results of using the slack minimization approach on the circuit test suite are shown in Table 6. In the table the three columns in the middle provide the power reduction in signal, logic, and clock dynamic power in percentage. The right-most column presents the overall power savings. As can be seen, this technique presents mixed results, with a few circuits obtaining positive results, most with negligible difference, and a few circuits even increasing in power consumption. The FM core contained no nets with only 1 or 2 levels of combinational logic and so was not applicable to this test run.

Individually, this technique proved the least successful and most difficult to work with. The clock tree paring, $\mathrm{N}$ terminal net colocation, and area minimization utilize placement constraints, effectively making the placement part of the PAR tools power savvy and balancing the work load of the PAR tools well between the placer and the router, and little to no growth in runtime operation of the PAR tools was observed. The slack minimization technique however utilizes 
timing constraints, effectively putting both the power optimization and original timing constraint work loads onto the router portion of the PAR tools. PAR runtime increased sharply using this technique and it was observed that even though slack was minimized on the specified nets, unspecified nets would often experience a corresponding increase in wire length. Tightening the slack on too many nets would also result in the original timing specifications to be unable to be met. While individually this technique did not yield good results, as we will see in Section 5 , this technique did prove useful when combined with the other techniques.

\section{COMBINED POWER OPTIMIZATION}

In the previous experiments, the four power optimization approaches are considered individually in order to determine the effects of the algorithm and learn more about power consumption, the underlying FPGA architecture, and the behavior of the COTS PAR tools. As we have observed, the clock paring technique yields good results, while the rest of the techniques provide mixed results. A more detailed analysis of the test circuits and our results shows that on a per net perspective, the clocks are the most dominant power consumers for all circuits in our test bench. Moreover, all of the techniques presented are complimentary, utilizing different constraint types, and can be combined together. So for the last experiment in our paper, we will consider clock tree paring to be a first order optimization that needs to be performed before we can truly measure the results of the second-order optimizations, $\mathrm{N}$-terminal net colocation, area minimization, and slack minimization. As all of the techniques are complimentary we will consider the case where all of the constraints are applied to simplify our discussion.

Table 7 shows the overall results for the combined optimization techniques, the additional power savings over the first-order optimization, and the total power saved for each circuit. As shown in the table, 5 out of 10 benchmark designs reach their maximum power reduction by using a combination of techniques. In the referencing of Table 2, the circuits which seemed to respond well to multiple optimizations, CRC, Conv, and Mem, are all logic power dominated circuits. Clock power dominated circuits saw little to no benefit from combining constraints. The final power reduction ranges from $2.9 \%$ to $19.4 \%$, and the average improvement is $10.2 \%$.

\section{HARDWARE VALIDATION RESULTS}

In this section we seek to validate that the results we have seen in the previous sections utilizing XPower and our LITE tools are realizable in the real world. However, the real world brings other constraints that further complicate matters. For starters, the Osiris FPGA hardware boards have a fixed FPGA device, the V2 6000. While S1, S2, and Mem from our test suite target this same device, S1 and S2 assume different bus and memory interfaces than our hardware, and the Mem kernel did not produce enough dynamic power to yield statistically stable data with the resolution of our A/D board and the current sensors in our testbed.
TABLE 7: Combined power optimization results.

\begin{tabular}{l|ccc}
\hline Design & $\begin{array}{l}\text { Combined } \\
\text { power } \\
\text { reduction }\end{array}$ & $\begin{array}{l}\text { Increase } \\
\text { over } \\
\text { clock } \\
\text { paring }\end{array}$ & $\begin{array}{l}\text { Max } \\
\text { power } \\
\text { saved } \\
(\mathrm{mW})\end{array}$ \\
\hline CRC & $6.7 \%$ & $0.8 \%$ & 2 \\
FM & $2.9 \%$ & $0.0 \%$ & 3 \\
VGA & $12.7 \%$ & $0.2 \%$ & 17 \\
USBF & $10.7 \%$ & $0.0 \%$ & 9 \\
PCI & $19.4 \%$ & $0.7 \%$ & 8 \\
Conv & $7.1 \%$ & $2.2 \%$ & 9 \\
DES3 & $8.6 \%$ & $0.0 \%$ & 12 \\
Mem & $3.3 \%$ & $2.6 \%$ & 21 \\
S1 & $10.7 \%$ & $0.0 \%$ & 27 \\
S2 & $19.4 \%$ & $0.0 \%$ & 116 \\
\hline
\end{tabular}

TABLE 8: Hardware power measurement results.

\begin{tabular}{l|ccc}
\hline $\begin{array}{l}\text { Design } \\
\text { description }\end{array}$ & $\begin{array}{l}\text { XPower } \\
\text { estimation } \\
(\mathrm{mW})\end{array}$ & $\begin{array}{l}\text { Hardware } \\
\text { result } \\
(\mathrm{mW})\end{array}$ & $\begin{array}{l}\text { XPower: } \\
\text { measure } \\
\text { ratio }\end{array}$ \\
\hline Baseline & 351 & 281 & 1.25 \\
Clock paring & 334 & 270 & 1.24 \\
Slack minimization & 352 & 286 & 1.23 \\
$\begin{array}{l}N \text {-terminal net } \\
\text { colocation }\end{array}$ & 354 & 280 & 1.26 \\
Area minimization & 342 & 270 & 1.26 \\
Combined & 333 & 268 & 1.24 \\
\hline
\end{tabular}

So for the purposes of this paper, we created a variant of the Conv circuit to be tested on the hardware. In this version, the Conv circuit was instanced 5 times in order to fill the device and achieve large enough power for measurement in our testbed.

The measurement results as well as the XPower estimation are shown in Table 8 . The table lists the power results of the unoptimized design (baseline), the power optimized designs that use a single power technique, and the combined technique power optimized design. The second column provides the dynamic power consumption estimated by the Xilinx XPower tool. The third column is the power number measured on hardware. The final column calculates the ratio of the software measured values to that of the hardware measured values. So, while XPower seems to report a consistently higher value than that measured on hardware, the ratio is nearly constant, approximately 1.24. Power optimizations measured in software carry over into hardware. Though the absolute power varies, the relative percentage of power decreased remains relatively constant between software and hardware.

\section{CONCLUSIONS}

In this paper, we present a variety of techniques that seek to reduce power by feeding power driven constraints into COTS 
PAR tools. These constraints seek to influence the FPGA implementation tools to place and route a user's design in a more power efficient manner. Four power optimization approaches are introduced in detail and are evaluated in Xilinx Virtex-II FPGA devices. The results show that the clock tree is the dominant dynamic power contributor and the clock tree paring approach is the most effective method to save power. The techniques are not mutually exclusive and clock tree paring can be combined with the other techniques to further reduce power. The average overall dynamic power savings on our test suite is $10.2 \%$. Though our experimentation has focused on the Xilinx Virtex-II architecture, these techniques are expected to have similar results on other FPGA devices as well.

\section{ACKNOWLEDGMENTS}

The authors thank Michael Wirthlin, Kevin Lundgreen, and Nathan Rollins of Brigham Young University for their assistance with JHDL/EDIF infrastructure. This research was performed under NASA AIST Grant NAG5-13516, Reconfigurable Hardware in Orbit (RHINO).

\section{REFERENCES}

[1] J. H. Anderson, F. N. Najm, and T. Tuan, "Active leakage power optimization for FPGAs," in Proceedings of the ACM/SIGDA International Symposium on Field Programmable Gate Arrays (FPGA '04), vol. 12, pp. 33-41, Monterey, Calif, USA, February 2004.

[2] M. French, "A power efficient image convolution engine for field programmable gate arrays," in 7th Annual International Conference on Military and Aerospace Programmable Logic Devices (MAPLD '04), Washington, DC, USA, September 2004.

[3] J. H. Anderson and F. N. Najm, "A novel low-power FPGA routing switch," in Proceedings of the IEEE Custom Integrated Circuits Conference (CICC '04), pp. 719-722, Orlando, Fla, USA, October 2004.

[4] E. Kusse and J. Rabaey, "Low-energy embedded FPGA structures," in Proceedings of the International Symposium on Low Power Electronics and Design, pp. 155-160, Monterey, Calif, USA, August 1998.

[5] J. H. Anderson and F. N. Najm, "Power-aware technology mapping for LUT-based FPGAs," in IEEE International Conference on Field-Programmable Technology (FPT '02), pp. 211218, Hong Kong, December 2002.

[6] N. Rollins and M. J. Wirthlin, "Reducing energy in FPGA multipliers through glitch reduction," in 7th Annual International Conference on Military Applications of Programmable Logic Devices (MAPLD '05), Washington, DC, USA, September 2005.

[7] J. Lamoureux and S. J. E. Wilton, "On the interaction between power-aware FPGA CAD algorithms," in IEEE/ACM International Conference on Computer-Aided Design (ICCAD '03), pp. 701-708, San Jose, Calif, USA, November 2003.

[8] L. Shang, A. S. Kaviani, and K. Bathala, "Dynamic power consumption in virtex-II FPGA family," in Proceedings of the ACM/SIGDA International Symposium on Field Programmable Gate Arrays (FPGA '02), pp. 157-164, Monterey, Calif, USA, February 2002.

[9] "Virtex-II Platform FPGAs: Complete Data Sheet," www. xilinx.com.
[10] Xilinx ISE Software Manual, www.xilinx.com.

[11] M. French, L. Wang, T. Anderson, and M. Wirthlin, "Post synthesis level power modeling of FPGAs," in IEEE Symposium on Field-Programmable Custom Computing Machines (FCCM '05), pp. 281-282, Napa, Calif, USA, April 2005.

Li Wang received the B.E. degree in electrical engineering from Tsinghua University, Beijing, China, in 1998, and the M.S. degree in electrical and computer engineering from the University of Maryland, College Park, in 2001, where she is currently pursuing the Ph.D. degree. She has been a Computer Systems Engineer since 2001 with the Information Sciences Institute, the University of Southern California working in Dynamic Systems Division. Her current research interests include lowpower FPGA, low-power computing systems, analog VLSI, and biomedical engineering especially in heart models.

Matthew French is a Project Leader at the Information Sciences Institute, University of Southern California, and leads research in application mapping to embedded computing systems, incorporating novel computing architectures, ruggedized environment constraints, and tool development. $\mathrm{He}$ has over 10 years experience in the field of adaptive computing systems and holds 3 FPGA-related patents. Prior to USC/ISI, he worked at Lockheed Sanders on a variety of communications and SIGINT platforms. He received the Masters of Engineering and Bachelors of Science degrees from Cornell University, in 1996.

Azadeh Davoodi received the B.S. degree in electrical and computer engineering from the University of Tehran, Iran, in 1999, and the M.S. degree from University of Maryland, College Park, in 2002, where she is currently a Ph.D. candidate. Her research interests include design automation issues for ASICs and FPGAs in deep submicron fabrication technologies, such as power optimization and interconnect modeling.

Deepak Agarwal received the B.Tech. degree in electrical engineering from Indian Institute of Technology (IIT), Kanpur, in 1999 and joined Texas Instruments (TI) as an IC Design Engineer. At TI, he was part of the team that successfully designed the C28X DSP core. In 2001, he joined Proceler Inc. Atlanta as a Senior Systems Engineer where he worked on design problems related to reconfigurable computing. Follow-

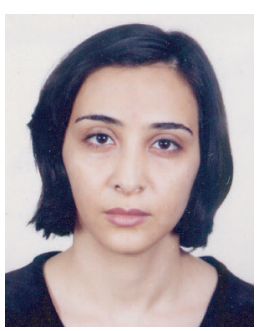
ing this, he enrolled at Virginia Polytechnic Institute and State University where he was a Graduate Research Assistant at the Configurable Computing Lab and received his M.S. degree in computer engineering in 2005. He is currently a Staff Hardware Engineer at National Instruments in the Distributed IO Group. His research interests include computer architecture, VLSI, reconfigurable computing, ASIC/FPGA design and testing.

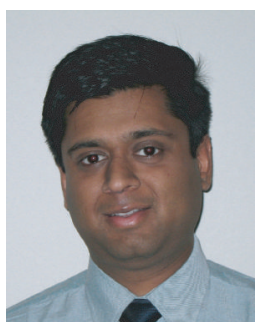

